\title{
COMPARISON OF CLINICAL OUTCOMES AFTER FEMTOSECOND ASSISTED LASER IN SITU KERATOMILEUSISAND IMPLANTABLE COLLAMER LENS IMPLANTATION FOR CORRECTION OF HIGH MYOPIA
}

\author{
Abdelmateen, E. ${ }^{(*)}$, Mostafa, E., Ammar, H. \& Abdallah, A. \\ Ophthalmology dept.., Faculty of Medicine, Sohag Univ., Sohag, Egypt \\ *E-mail: elshimaa.moussa@yahoo.com
}

\begin{abstract}
Purpose: To compare efficacy, safety and stability of Femtosecond assisted LASIK and implantable collamer lens implantation in correction of high myopia. Methods: This study was nonrandomized comparative prospective consecutive interventional study. It included 74 eyes with high myopia ( $\geq-6$ Ds) of 40 patients attended to the outpatient ophthalmic clinic of Sohag university hospitals from the period from Jan.2016 to Jan.2017. The patients were divided into two groups depending on: Degree of myopia, corneal thickness and corneal tomography. The first group (34 eyes) of 20 patients were subjected to implantable collamer lenses implantation and the second group (40 eyes) of 40 patients were subjected to femtosecond assisted LASIK. Results: Group one (ICL group) showed UCVA preoperative was (1.90 \pm 0.29$)$ and UCVA postoperative was $(0.27 \pm 0.21)$ with $\left(p\right.$-value $\left.<0.000^{*}\right)$. BCVA preoperative was $(0.526 \pm 0.272)$ and that of BCVA postoperative was $(0.217 \pm 0.128)$ with ( $p$-value $\left.<0.001^{*}\right)$, which means that BCVA postoperative was better than what was expected from the BCVA preoperative. Spherical error decreased from $(-13.576 \pm 3.945)$ preoperative to $(-0.0385 \pm 0.821)$ postoperative with $\left(p\right.$-value $\left.<0.000^{*}\right)$. Cylindrical error preoperative was $(-1.134 \pm 0.617)$ and postoperative was $(-0.352 \pm 0.250)$ with $\left(p\right.$-value $\left.<0.000^{*}\right)$.Spherical equivalent preoperative was $(-15.173 \pm$ $4.079)$ and that of postoperative was $(-0.269 \pm 0.787)$ with $\left(p\right.$-value $\left.<0.000^{*}\right)$. Pachymetry preoperative was $(518.153 \pm 22.981)$ and postoperative was (521 \pm 22.304$)$ with ( $p$-value $<0.140)$. Group two (Femtosecond assisted LASIK) UCVA preoperative was $(1.42 \pm 0.27)$ and UCVA postoperative was $(0.366 \pm 0.21)$ with $\left(p\right.$-value $\left.<0.000^{*}\right)$. There wasn't significant difference between BCVA preoperative and postoperative. Spherical error preoperative was $(-8.398 \pm 1.733)$ and postoperative $(-0.796 \pm 1.186)$ with $\left(p\right.$-value $\left.<0.000^{*}\right)$. Cylinder error mean value preoperative was ($1.648 \pm 0.838)$ corrected to $(-0.765 \pm 0.423)$ with $(p$-value $<0.005)$. Spherical equivalent preoperative was $(-9.213 \pm 1.899)$ and that of postoperative is $(-0.935 \pm 1.11)$ with $\left(p\right.$-value $\left.<0.000^{*}\right)$. Pachymetry preoperative was $(535.111 \pm 26.33)$ and postoperative $(419.88 \pm 43.063)$ with ( $p$ value $<0.000^{*}$. Conclusion: Both implantable collamer lens and Femtosecond assisted LASIK proved high efficacy and safety but implantable collamer lens showed more stability for high myopic patients.
\end{abstract}

Keywords: High myopia, Femtosecond, Excimer laser, Implantable collamer lens, Efficacy, Safety, Stability.

\section{Introduction}

Myopic eye bends the coming light too much, which means that the light comes to a focus point before it reaches the retina. When moving the target object closer to the eye, this changes the focusing of the light and the object is then in 
focus on the retina and therefore looks clear. Myopic can be classified into mild myopia includes powers up to -3.00 diopters (D), moderate myopia, values of -3.00 to $-6.00 \mathrm{D}$ and high myopia is usually myopia over -6.00D [1]. Femtosecond (FS) laser is an infrared laser has a wavelength of $1053 \mathrm{~nm}$. It works by production of photo disruption or photo ionization of the optically transparent tissue such as the cornea [1]. Phakic intraocular lenses (PIOLs) are alternative treatment for ametropia correction among various refractive ranges. Fast visual recovery, high efficacy, predictability and stability of visual quality, preservation of accommodation, and reversibility are several advantages that have been attributed to PIOL implantation [2]. The Visian Implantable Collamer Lens (ICL; STAAR Surgical Co, Monrovia, California) is approved by the United States Food and Drug Administration (FDA) for the treatment of moderate to high myopia. The trade-

\section{Patients and methods}

Forty individuals (34 eyes) subjected to implantable collamer lens implanttation surgery and (40 eyes) subjected to femtosecond assisted Lasik surgery , aged 27-33 years who attended to the outpatient ophthalmic clinic of Sohag university hospitals from the period from Jan.2016 to Jan.2017.Group one (ICL) had Spherical refractive errors mean value $-13.576 \mathrm{D}( \pm 3.945)$ with astigmatism $-1.134 \mathrm{D}( \pm 0.617)$. The patients were with clear intraocular media and had no ocular pathology. Group two (Femto second assisted Lasik) had Spherical refractive errors mean value $-8.398( \pm 1.733)$ with astigmatism $-1.648( \pm 0.838)$. The patients were with clear intraocular media and no had no ocular pathology. The tenets of the declaration of Helsinki were followed. Informed consent was obtained from each participant after verbal and written explanations of the nature and possible consequences of the study were provided .The study protocol received institutional review board appr- name of lens material is Collamer, is a hydrophilic collagen-polymer combination with a water content of $34 \%$ and a refractive index of 1.45 [3]. Although the ICL offers outstanding advantages, there have been reports in the literature of postoperative complications associated with high and low vaults of the ICL over the crystalline lens. Low vault leads to mechanical contact with the crystalline lens or inadequate circulation of the aqueous, which is responsible for a high incidence of anterior capsular opacification and cataract formation [4,5]. Conversely, marked high vault of the lens causes mechanical contact between the ICL and the iris, resulting in inflammation, high intraocular pressure, angle-closure glaucoma and pigment dispersion syndrome [6]. Aim of the work is to compare efficacy, safety and stability of Femtosecond assisted LASIK and implantable collamer lens implantation in correction of high myopia.

oval. The Visian ICL is a plate-haptic single-piece intraocular lens, which is a flexible. It is folded and implanted in the posterior chamber via a $2.8-3.2 \mathrm{~mm}$ corneal incision. It has a high degree of biocompatibility, permeability of gases and metabolites, and absorption of ultraviolet radiation. In this study, the ICL V4c lens designs phakic IOL were used. The ICL $\mathrm{V} 4 \mathrm{c}$ has a width of a $6.00 \mathrm{~mm}$ and is found in four sizes $(12.1,12.6,13.2$ and $13.7 \mathrm{~mm}$ in length). It has an optic zone diameter is $4.9-5.8 \mathrm{~mm}$, with a spherical power of -0.50 up to -18.00 DS and a cylindrical power range of +0.50 up to +6.00 DC. ICL power were calculated by the manufacturer (STAAR Surgical) using a modified vertex formula. The variables in the formula included preoperative manifest spherical and cycloplegic refractions, keratometric power, central corneal thickness and central anterior chamber depth (ACD, Pentacam, measured from the corneal endothelium to the anterior lens). The size (length) of the 
implanted ICL was determined based on the patient's WTW and ACD. For the ICL V4c, the sizes (lengths) of 12.1, 12.6, 13.2 and $13.7 \mathrm{~mm}$ were equal to the ICL V4 sizes (lengths) of 11.5, 12.0, 12.5 and $13.0 \mathrm{~mm}$, respectively [7]. ICL Surgical Procedure, on the day of surgery, patients apply dilating and cycloplegic agents, after which an anesthetic is applied to the operative eye. After placement of viscoelastic into the anterior chamber, ICL insertion through a small 3-mm clear corneal incision. The lens was injected through the incision into the anterior chamber (STAAR Micro STAAR injector) and is allowed to slowly unfold. Distal and proximal footplates are tucked under the iris with a modified intraocular spatula. The ICL is positioned correctly in the center of the pupillary zone before intraocular miotic is used to decrease pupil size. Any remaining viscoelastic is scrupulously irrigated out of the anterior chamber with balanced salt solution (BSS) [8], fig. (1). Flap creation using (FS), the suction ring is centered over the pupil and suction is applied once the proper centration of the ring has been ensured. The docking procedure is then initiated while the suction ring is kept parallel to the eye. An applanating glass contact lens is used to stabilize the globe and to flatten the cornea. It is important to achieve complete applanation of the cornea to avoid an incomplete flap or other flap related complications. Once the laser's computer has confirmed centration, the surgeon administers the FS laser treatment. Each pulse of the laser ene- rates free electrons and ionized molecules leading to formation of microscopic gas bubbles dissipating into surrounding tissue. Multiple pulses are applied next to each other to create a cleavage plane and ultimately the LASIK flap. Suction is then released. A spatula is passed across the flap starting from the hinge and sweeping inferiorly to lift the flap for starting Excimer laser ablation [9-12]. Efficacy by definition is the percentage of eyes with uncorrected visual acuity (UCVA) of 20/20 and 20/40 (2) Efficacy index, which is the ratio of the mean postoperative UCVA to the mean preoperative BCVA (i.e. mean postop. UCVA/mean preop. BCVA). (This is most easily calculated by converting the values of geometric mean acuities to decimal values) This measure is particularly useful in describing outcomes of high myopia when the preoperative BCVA is worse than 20/20 [13]. Safety can be defined as number and percentage of eyes losing two or more lines of best spectacle corrected visual acuity BCVA. (2) Safety index, which is the ratio of mean BCVA over mean preop. BCVA (i.e. Mean postop. BCVA/ mean preop. BCVA) this is most easily calculated by converting the values of geometric mean acuities to decimal values [13]. Stability is the number and percentage of eyes with a change of spherical equivalent of manifest refraction $\geq 1.00 \mathrm{D}$ within a specified interval, the recommended minimal interval is 6 months [13].

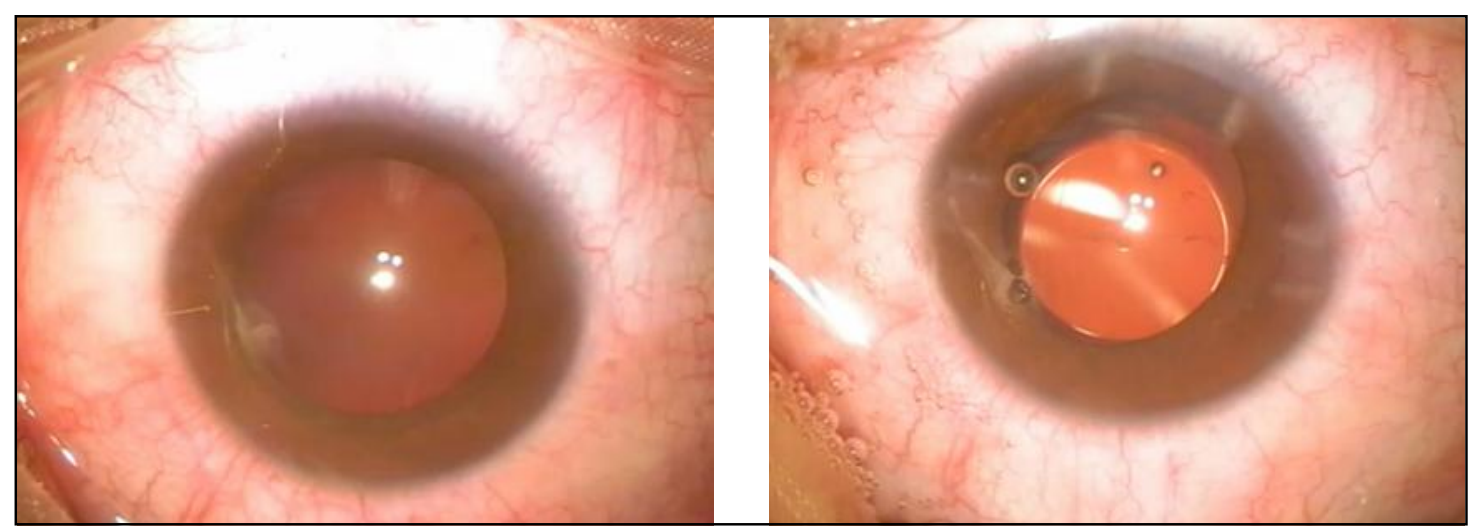

Figure (1) Shows pre and post ICL implantation 


\section{Statistical analysis}

Statistical analysis was performed using SPSS version 16 (IBM, USA). An independent samples t-test was used to compare mean values of measured para-

\section{Results}

Group one (ICL) showed UCVA preoperative was $(1.90 \pm 0.29)$ and UCVA postoperative was $(0.27 \pm 0.21)$ with (pvalue $<0.000^{*}$ ). BCVA preoperative was $(0.526 \pm 0.272)$ and that of BCVA postoperative was $(0.217 \pm 0.128)$ with (pvalue $<0.001^{*}$ ), which means that BCVA postoperative was better than what was expected from the BCVA preoperative. Spherical error decreased from $(-13.576 \pm$ $3.945)$ preoperative to $(-0.0385 \pm 0.821)$ postoperative with ( $\mathrm{p}$-value $<0.000 *$ ). Cylindrical error preoperative was $(-1.134 \pm$ $0.617)$ and postoperative was $(-0.352 \pm$ 0.250 ) with (p-value $<0.000 *)$.Spherical equivalent preoperative was $(-15.173 \pm$ 4.079) and that of postoperative was ($0.269 \pm 0.787)$ with (p-value $\left.<0.000^{*}\right)$. Pachymetry preoperative was $(518.153 \pm$ $22.981)$ and postoperative was $(521 \pm$ 22.304) with (p-value <0.140). Group two (Femtosecond assisted Lasik) showed UCVA preoperative was $(1.42 \pm 0.27)$ and UCVA postoperative was $(0.366 \pm 0.21)$ with (p-value $\left.<0.000^{*}\right)$. There wasn't significant difference between BCVA preoperative and postoperative. Spherical error preoperative was $(-8.398 \pm 1.733)$ and postoperative $(-0.796 \pm 1.186)$ with ( $\mathrm{p}$-value $\left.<0.000^{*}\right)$. Cylinder error mean value preoperative was $(-1.648 \pm 0.838)$ corrected to $(-0.765 \pm 0.423)$ with (pvalue $<0.005)$.Spherical equivalent preoperative was $(-9.213 \pm 1.899)$ and that of postoperative is $(-0.935 \pm 1.11)$ with $(\mathrm{p}-$ value $\left.<0.000^{*}\right)$. Pachymetry preoperative meters. Pearson's correlation coefficient was used to evaluate the correlation between quantitative variables.

was (535.111 \pm 26.33$)$ and postoperative $(419.88 \pm 43.063)$ with (p-value $<0.000 *)$. There wasn't significant difference in UCVA, BCVA, spherical error, cylindrical error or spherical equivalent preoperatively between both groups. There wasn't significant difference in UCVA and BCVA in both groups. Spherical error in group1 $(0.321 \pm 0.590)$ and group2 $(-0.75 \pm 0.31)$ with (p-value $<0.001 *)$. Cylindrical error in group1 $(-0.392 \pm 0.318)$ and in group2 $(-0.750 \pm 0.204)$ with ( $\mathrm{p}$-value $\left.<0.00^{*}\right)$. Spherical equivalent mean value of group 1 $(0.0000 \pm 0.433)$, in group2 (-1.392 \pm 0.748$)$ (p-value $<0.001 *)$. There wasn't significant difference in pachymetry in group1 pre and postoperative while there was significant difference in pachymetry of group 2 comparing pre and postoperative data. There was significant difference in pachymetry postoperative in between both groups with (521 \pm 22.304$)$ for group1 and $(419.88 \pm 43.1)$ with (p-value $\left.<0.00^{*}\right)$. However, although there is significant difference in pachymetry postoperative between both groups due to central ablation in group 2, but still the average pachymetric reading above 400um which is attributed to the use of the corneal thickness saving technique (Femto second assisted LASIK technique), tab. (1). Regarding complications in this study, it can be divided into intraoperative and postoperative complications for both groups, tab. (2).

Table (1) Postoperative results for both procedures

\begin{tabular}{|l|c|c|c|} 
& Group 1 (ICL) (n=34) & $\begin{array}{c}\text { Group 2 (Femto LASIK) } \\
(\mathbf{n = 4 0})\end{array}$ & P-value \\
\hline UCVA & $0.27( \pm 0.21)$ & $0.366( \pm 0.21)$ & $\mathbf{0 . 3 0 9}$ \\
\hline BCVA & $0.217( \pm 0.128)$ & $0.32( \pm 0.21)$ & $\mathbf{0 . 0 5 7}$ \\
\hline Spherical error & $-0.038( \pm 0.821)$ & $-0.79( \pm 1.18)$ & $\mathbf{0 . 0 9 9}$ \\
\hline
\end{tabular}




\begin{tabular}{|l|l|l|l|}
\hline Cylindrical error & $-0.352(0.250)$ & $-0.765( \pm 0.42)$ & $\mathbf{0 . 0 0 2} *$ \\
\hline Spherical equivalent & $-0.269( \pm 0.787)$ & $-0.935( \pm 1.12)$ & $\mathbf{0 . 1 3 8}$ \\
\hline Pachymetry & $521( \pm 22.304)$ & $419.88( \pm 43.1)$ & $\mathbf{0 . 0 0} *$ \\
\hline
\end{tabular}

Table (2) Intra and postoperative complications in the study

\begin{tabular}{|c|c|c|}
\hline 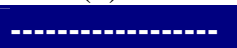 & Group 1 & Group 2 \\
\hline Intra operative & $\begin{array}{l}\text { - One eye ICL implanted upside down } \\
\text { with partially torn haptic during } \\
\text { manipulations for removal and } \\
\text { reinsertion }\end{array}$ & $\begin{array}{l}\text { - Three eyes showed decentered abla- } \\
\text { tions detected during follow up, fig. } \\
\text { (2) }\end{array}$ \\
\hline Postoperative & $\begin{array}{l}\text { - One eye developed cataract, fig. (3). } \\
\text { - One eye developed postoperative iritis } \\
\text { which can be attributed to the mani- } \\
\text { pulations made to remove and reinsert } \\
\text { the lens intraoperative } \\
\text { - Three eyes were steroid responders } \\
\text { and IOP improved by cessation of } \\
\text { steroids. }\end{array}$ & $\begin{array}{l}\text { - One eye showed DLK which was } \\
\text { improved by steroids, fig. (4). }\end{array}$ \\
\hline
\end{tabular}

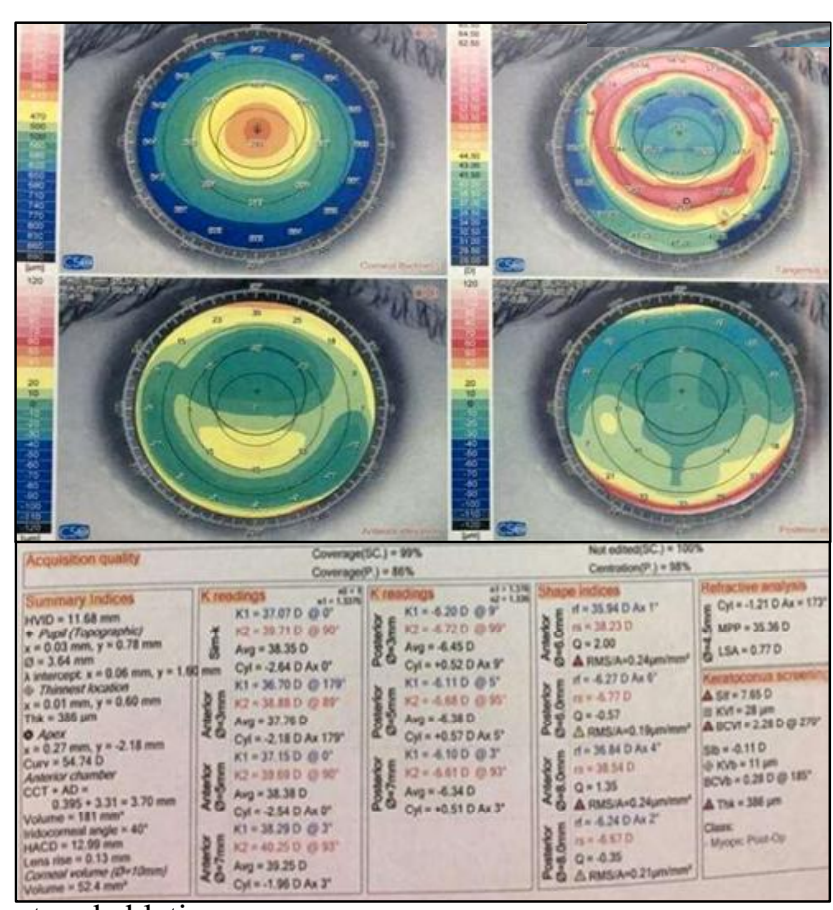

Figure (2) Shows decentered ablation

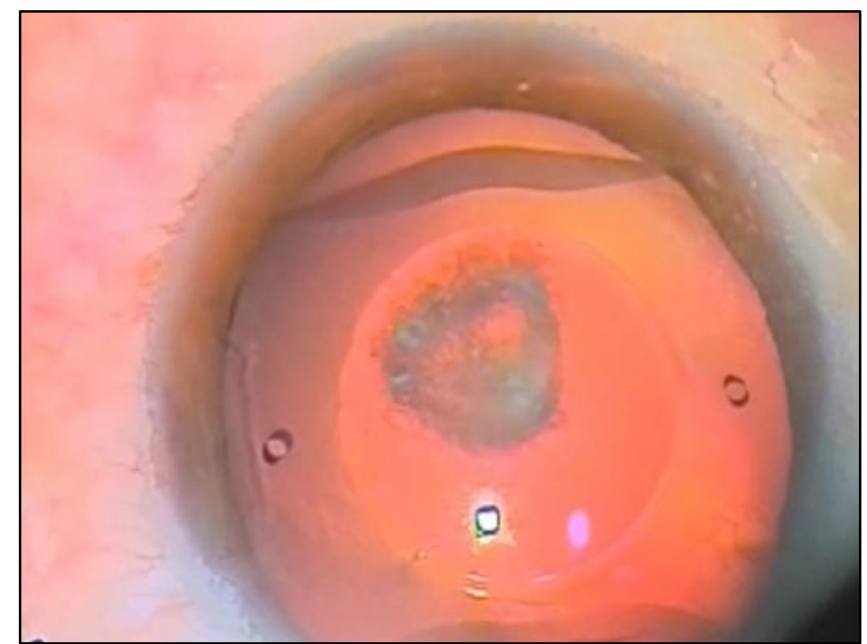

Figure (3) Shows complicated anterior sub capsular cataract 


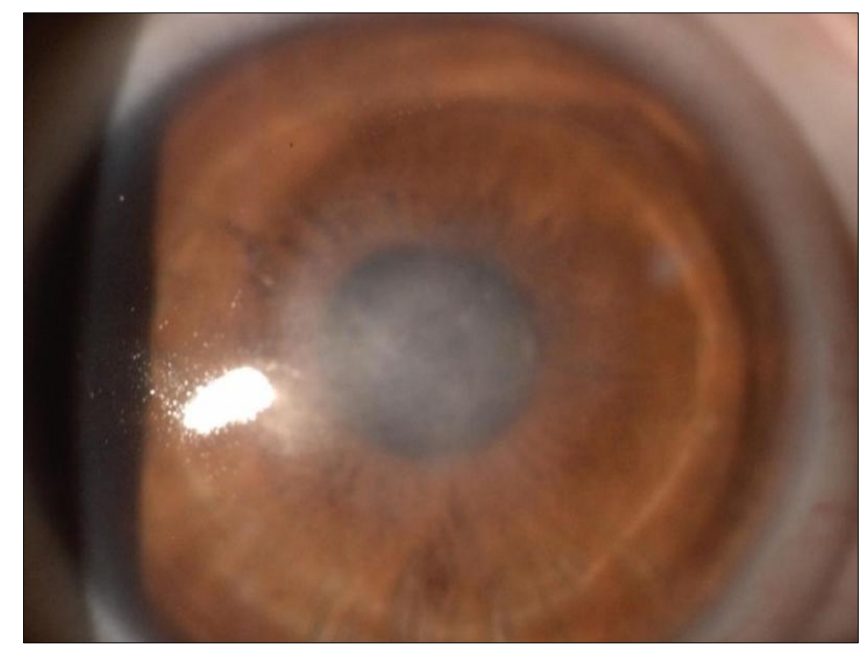

Figure (4) Shows postoperative DLK

Table (3) Postoperative efficacy and safety of both groups

\begin{tabular}{|l|c|c|}
\hline \multicolumn{1}{|c|}{} & $\begin{array}{c}\text { Group one(ICL) } \\
(\mathbf{n = 3 4 )}\end{array}$ & $\begin{array}{c}\text { Group two (Femtosecond Lasik) } \\
(\mathbf{n = 4 0})\end{array}$ \\
\hline Efficacy & 0.95 & 1.01 \\
\hline Safety & 1.9 & 0.98 \\
\hline
\end{tabular}

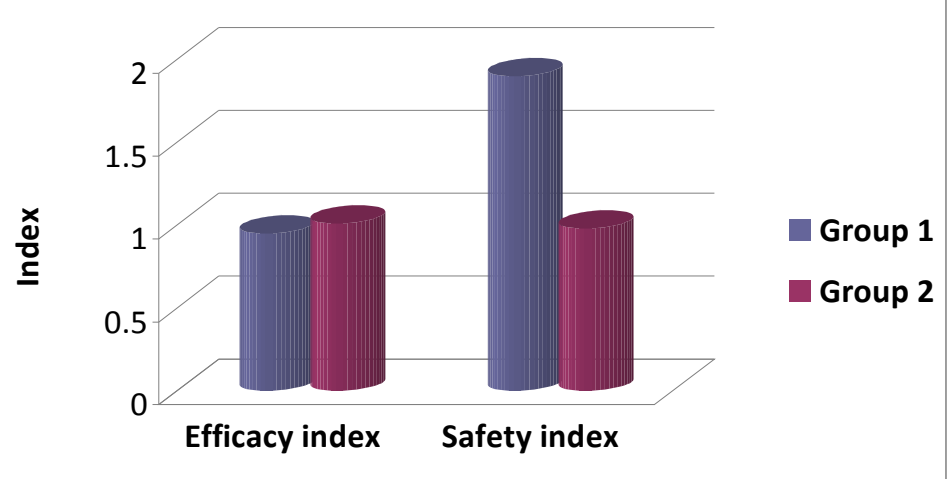

Figure (5) Shows both groups showed high efficacy, but Group1 showed more safety (Groupl showed $100 \%$ stability while group 2 showed $71.4 \%$, as shown in the below graph)

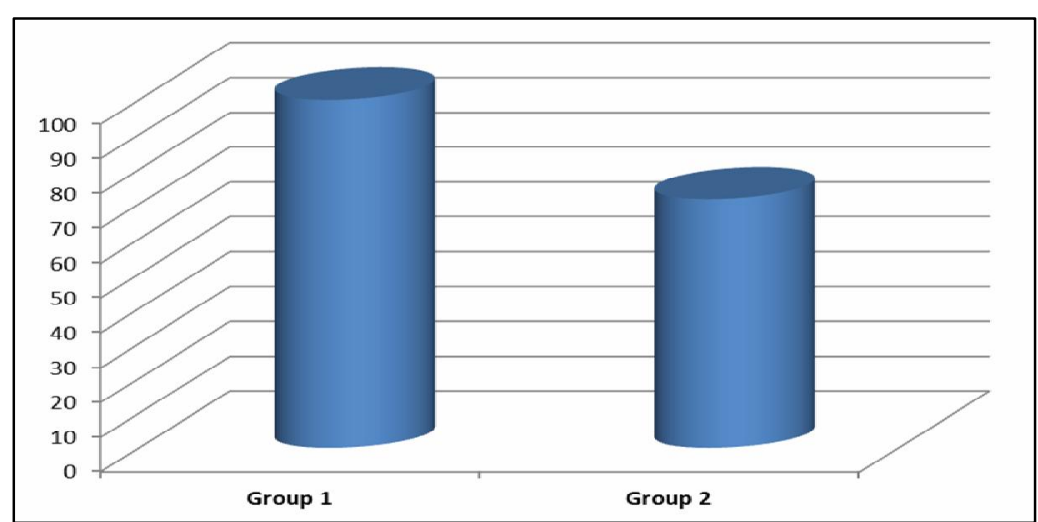

Figure (6) Shows graph showing difference in stability between both groups

\section{Discussion}

The aim of this study was to evaluate the visual outcomes of femtosecond LASIK in comparison with implantable collamer lenses for correction of high myopia regarding visual acuity, efficacy, safety and stability. The patients were divided into two groups. The first group was subjected to implantable collamer 
lenses implantation and the second group subjected to femtosecond assisted LASIK. Excimer laser surgeries proved effectiveness for myopia correction, but they have complications such as myopic regression and ectasia which were reported in eyes with high myopia [14,15]. It is proved that Phakic IOL implantation has predictability in correction of high myopia [16, 17]. Implantation of PIOL can induce complications such as cataract, lens dislocation and elevation of intraocular pressure. Therefore, assessing visual and outcomes of PIOL is helpful when selecting the more appropriate, safe, stable and effective procedure to correct high myopia, especially when the patients have an overlapping range of both procedures [18]. This study showed excellent refractive and visual outcomes with both used procedures of ICL implantation and Femtosecond assisted LASIK. The study showed that with a pupillary diameter 3-mm, VA values are improved in $100 \%$ of cases which can be up to $(0.1$ $\log$ MAR or more) after 6 months follow up $(p<0.000 *)$. There is agreement between our results and results of Paul and Taylor who studied refractive outcomes and safety of the implantable collamer lens in young low to moderate myopes. Their study was retrospective study performed by chart review of (104 eyes) with 50 months follow up period [19]. The results of group two (Femtosecond assisted LASIK group) from the visual quality point of view with a pupillary diameter 4-mm, VA values improved in $100 \%$ of cases which is up to $(0.1 \log$ MAR or more) after 6 months follow up, evaluated $\left(\mathrm{p}<0.00^{*}\right)$. This result is consistent with that of Cari Perez-Vives et al [20]. Both groups showed high efficacy and safety, but group one (ICL) showed more stability during the follow up period than group two (Femtosecond Lasik), fig. s. $^{5} \&$ 6), tab. (3). These finding were consistent with that of Xun Chen et, al. who studied contralateral eye comparison of the long-term visual quality and stability between implantable collamer lens and laser refractive surgery for myopia. Their study conducted on 52 eyes of
26 high-myopia anisometropia patients who were suitable for surgical treatment. In each patient, the higher-myopia eye was implanted with ICL and the lower myopia eye was treated with LRS. The patients were followed for 3 years [21]. Regarding intraoperative complications of Group 1 are one eye ICL implanted upside down with partially torn haptic during manipulations for removal and reinsertion. It is a rare complication of ICL implantation which is mentioned by Amar Agarwal and Kumar, who studied Visco-cannula assists in rein version of phakic lens [22], and that of Group 2 are three eyes showed decentered ablations detected during follow up. This complication is stated by Hiroko Biessen-Miyajima, a book of cataract and refractive surgery [23]. Postoperative complications of Group1 are one eye developed cataract. This complications is also mentioned by Seyed Javad Hashemian, MD, et al studied the outcomes and complications of ICL and toric ICL for the correction of high myopia with and without astigmatism, whose study involved 95 eyes and followed up for one year [24]. One eye developed postoperative iritis which can be attributed to the manipulations made to remove and reinsert the lens intraoperative. Three eyes were steroid responders and IOP improved by cessation of steroids. This complication is encountered by Sirish Senthil, et al. who studied etiology and management of raised intraocular pressure following posterior chamber phakic intraocular lens implantation in myopic eyes. They studied 638 eyes between 2009 and 2015 [25]. Postoperative complication of Group 2 is one eye showed DLK which was improved by steroids. This complication was encountered by Ayad A. Farjo, MD, et al. who studied femtosecond lasers for LASIK flap creation, a report by the American academy of ophthalmoology [26]. In conclusion both Implantable collamer lens implantation and femtosecond assisted Lasik for high myopia proved high efficacy and safety but ICL showed more stability during the follow up period. 


\section{Conclusion}

Both implantable collamer lens and Femtosecond assisted LASIK proved high efficacy and safety but implantable collamer lens showed more stability for high myopic patients

\section{References}

1. Chung S., Mazur E. Surgical applications of femtosecond lasers. $\boldsymbol{J}$. of Biophotonics. 2009; 2 (10): 557572.

2. Sanders D., Doney K., Poco M. United states food and drug administration clinical trial of the implantable collamer lens (ICL) for moderate to high myopia: Three-year follow-up. Ophthalmology. 2004; 111 (9): 1683-1692.

3. Lovisolo C., Reinstein D. Phakic intraocular lenses. Survey of ophthalmology. 2005; 50 (6): 549-587.

4. Sanders D., Vukich J., Doney K., Gaston M. U.S. food and drug administration clinical trial of the implantable contact lens for moderate to high myopia. Ophthalmology. 2003; 110 (2): 255-266.

5. Schmidinger G., Lackner B., Pieh S., Skorpik C. Long-term changes in posterior chamber phakic intraocular collamer lens vaulting in myopic patients. Ophthalmology. 2010; 117 (8):1506-1511.

6. Lackner B., Pieh S., Schmidinger G., Hanselmayer G., Dejaco-Ruhswurm I., Funovics M., et al. Outcome after treatment of ametropia with implantable contact lenses. Ophthalmology. 2003; 110 (11): 2153-2161.

7. Chen X., Miao H., Naidu R., Wang X., Zhou X. Comparison of early changes in and factors affecting vault following posterior chamber phakic implantable collamer lens implantation without and with a central hole (ICL V4 and ICL V4c). BMC Ophthalmology. 2016; 16 (1): 161.

8. Sanders D., Vukich J. Comparison of implantable contact lens and laser assisted in situ keratomileusis for moderate to high myopia. Cornea. 2003; 22 (4): 324-331.

9. Durrie D., Kezirian G. Femtosecond laser versus mechanical keratome flaps in wavefront-guided laser in situ keratomileusis: prospective contralateral eye study. J. of Cataract and Refractive Surgery. 2005; 31 (1): 120-126.

10. Slade S. The use of the femtosecond laser in the customization of corneal flaps in laser in situ keratomileusis. Current Opinion in Ophthalmology. 2007; 18 (4): 314-317.

11. Soong H., Malta J. Femtosecond lasers in ophthalmology. Am J. Ophthalmol. 2009; 147 (2): 189-97.e2.

12. Kymionis G., Kankariya V., Plaka A., Reinstein D. Femtosecond laser technology in corneal refractive surgery: a review. J. of Refractive Surgery 2012; 28 (12): 912-920.

13. Koch D., Kohnen T., Obstbaum S., Rosen E. Format for reporting refractive surgical data. $\boldsymbol{J}$. of Cataract and Refractive Surgery. 1998; 24 (3): 285-287.

14. Krueger R., Talamo J., McDonald M., Varnell R., Wagoner M., McDonnell P. Clinical analysis of excimer laser photorefractive keratectomy using a multiple zone technique for severe myopia. Am. J. of Ophthalmology. 1995; 119 (3): 263274.

15. Stulting R., Carr J., Thompson K., Waring G., Wiley W., Walker J. Complications of laser in situ keratomileusis for the correction of myopia. Ophthalmology. 1999; 106 (1): 13-20.

16. Igarashi A., Kamiya K., Shimizu K., Komatsu M. Visual performance after implantable collamer lens implantation and wavefront-guided laser in situ keratomileusis for high myopia. Am. J. of Ophthalmology. 2009; 148 (1): 164-70.e1.

17. Sanders D., Vukich J. Comparison of implantable contact lens and laser assisted in situ keratomileusis for moderate to high myopia. Cornea. 2003; 22 (4): 324-331. 
18. Shin J., Ahn H, Seo K., Kim E., Kim T. Comparison of higher order aberrations after implantable Collamer Lens implantation and wavefrontguided LASEK in high myopia. $\boldsymbol{J}$. of Refractive Surgery 2012; 28 (2): 106-111.

19. Dougherty P., Priver T. Refractive outcomes and safety of the implantable collamer lens in young low-tomoderate myopes. Clin Ophthalmol. 2017; 11: 273-277.

20. Perez-Vives C., Albarran-Diego C., Garcia-Lazaro S., Ferrer-Blasco T., Montes-Mico R. Implantable collamer lens and femtosecond laser for myopia: comparison using an adaptive optics visual simulator. Arquivos Brasileiros de Oftalmologia. 2014; 77 (2): 103-109.

21. Chen X., Guo L., Han T., Wu L., Wang X., Zhou X. Contralateral eye comparison of the long-term visual quality and stability between implantable collamer lens and laser refractive surgery for myopia. Acta Ophthalmologica. 2019; 97 (3): e471-e478

22. Amar Agarwal M., Kumar D. Viscocannula assists in reinversion of phakic lens. Ocular Surgery News. 2009; 20 (4): 25.

23. Bissen-Miyajima $\mathrm{H}$. Complications of excimer laser surgery. Cataract and Refractive Surgery. 2006; 22 (7): 647655.

24. Hashemian S., Bigzadeh F., Foroutan A., Tajoddini S., Ghaempanah M., Jafari M. Outcomes and complications of implantable collamer lens and toric implantable collamer lens for the correction of high myopia with and without astigmatism (One Year Prospective Study). Iranian J. of Ophthalmology. 2013; 25 (1): 8.

25. Senthil S., Choudhari N., Vaddavalli P., Murthy S., Reddy J., Garudadri C. Etiology and management of raised intraocular pressure following posterior chamber phakic intraocular lens implantation in myopic eyes. PloS One. 2016; 11 (11): e0165469.

26. Farjo A., Sugar A., Schallhorn S., Majmudar P., Tanzer D., Trattler W., et al. Femtosecond lasers for LASIK flap creation: a report by the American academy of ophthalmology. Ophthalmology. 2013; 120 (3): e5e20. 